\title{
SER JOVEN HOY EN ESPAÑA. DIFICULTADES PARA EL ACCESO AL MUNDO DE LOS ADULTOS
}

\author{
BEING YOUNG NOWADAYS IN SPAIN. DIFFICULTIES IN ACCESSING TO \\ ADULTS WORLD
}

\author{
José Manuel García Moreno \\ Rafael Martínez Martín \\ Universidad de Granada. España/Spain \\ josemgar@ugr.es \\ rmmartin@ugr.es
}

Recibido/Received: 28/08/2012

Modificado/Modified: 27/09/2012

Aceptado/Accepted: 08/10/2012

\section{RESUMEN}

El interés por el estudio de los jóvenes españoles ha vuelto con fuerza desde el inicio de la crisis económica. No hay comparecencia pública en la que no se hable del trinomio joven -desempleoemancipación. Este nos obliga a tratar de explicar no solo las consecuencias directas del desempleo para los jóvenes, sino las consecuencias sociales que, la no transición de estos hacia el mundo de los adultos, tendrá a medio plazo para el conjunto de la sociedad española. El Estado de Bienestar se mantiene, entre otros elementos, con la fuerza del trabajo de los adultos pero, si nuestros jóvenes no pueden llegar a ser adultos, la pregunta es evidente ¿qué puede terminar pasando? En este trabajo debatimos sobre este hecho, sobre la emancipación, sobre la independencia, sobre los recursos necesarios para pasar al mundo adulto y sobre los escenarios que se pueden estar abriendo en la sociedad española.

\section{PALABRAS CLAVE}

Juventud, emancipación, transición, exclusión social, ciudadanía.

\section{SUMARIO}

1. Introducción. 2. La emancipación como factor de integración o exclusión social en el mundo adulto. 3. Los recursos de la emancipación. 3.1. La actividad productiva laboral como factor de independencia económica. 3.2. La vivienda propia como factor de emancipación. 4. Dependencia versus independencia. 5. Conclusiones. Bibliografía

\footnotetext{
ABSTRACT

The interest in the study of Spanish youth is with us from the beginning of the economic crisis. There is not public hearing where the trinomial young -unemployment- emancipation is not mentioned. This forces us to try to explain not only the direct consequences of unemployment for young people, but the social consequences which, the non transition of these into the adult world will have in the medium term for the whole Spanish society. The welfare state is maintained, among other things, by the power of adult work but if our young people can not become adults, the obvious question is what can end up happening? In this paper we discuss this fact, emancipation, independence, the resources required to move into the adult world and scenarios that can be opened in the Spanish society.
} 


\section{KEYWORDS}

Youth, emancipation, transition, social exclusion, citizenship.

\section{CONTENTS}

Introduction. 2. Emancipation as an integration or social exclusion factor in the adults world. 3. Emancipation resources. 3.1. Labor productive activity as an economic independence factor. 3.2. Homeownership as an emancipation factor. 4. Dependence versus independence. 5. Conclusions

\section{INTRODUCCIÓN}

Desde finales de 2007, cuando los síntomas de la crisis económica internacional comenzaban a reflejarse en el contexto español, el debate en torno al futuro de la juventud de nuestro país volvió con fuerza, por un lado, al escenario político y, por otro, al escenario de la academia. Es decir, si bien las tasas de desempleo de los jóvenes españoles siempre han estado por encima de la media de nuestro contexto europeo, el crecimiento de este indicador de forma alarmante desde ese año ponía sobre la mesa del debate ya no sólo las dificultades de inserción laboral sino, además, y esa es nuestra hipótesis fundamental, la propia inserción social de esa juventud que, por esta vía y poco a poco, puede acabar perdiendo su rol de ciudadano.

En este artículo presentamos algunas conclusiones de lo que es un trabajo más profundo que venimos desarrollando desde el año 2007, en el que hablamos de cómo la no emancipación y la no adquisición de los recursos propios de la adultez puede desembocar en la pérdida del referente social que supone desempeñar el rol de adulto.

Esta investigación es tanto de tipo cuantitativo como cualitativo. En el primer caso, se analiza, desde los inicios señalados, todos los estudios que van apareciendo y construyendo una base longitudinal que permita interpretar este fenómeno de forma más certera. En el segundo caso, la investigación cualitativa, se desarrolla con entrevistas en profundidad a jóvenes de entre 18 y 35 años que, analizadas con la técnica del análisis del discurso, permiten ampliar las hipótesis para tratar de contrastarlas con los datos de corte cuantitativo. En este artículo se presentan algunas referencias procedentes de ambas metodologías.

\section{LA EMANCIPACIÓN COMO FACTOR DE INTEGRACIÓN O EXCLUSIÓN SOCIAL EN EL MUNDO ADULTO}

Parsons (1998:45) afirmaba que "uno de los rasgos esenciales de nuestro sistema familiar es que, al alcanzar la madurez, los hijos han de emanciparse de sus familias de orientación y abrirse camino en el mundo por sí mismos, en vez de hacerlo en el seno del grupo de parentesco originario", en una visión profundamente funcional de la transición a la edad adulta, dentro de lo que serían los marcos consensuales de reproducción social. Sin embargo, y como todos conocemos en el caso español, no todo es tan aparentemente lineal como señalaba el padre del estructural-funcionalismo. Lo más habitual a la hora de interpretar la dimensión social de la juventud es considerar a ésta "como un período en el que se pasa de la vida dependiente propia de la niñez a las formas de independencia propia de los adultos" (Garrido y Requena, 1997:15). Cuando nos referimos a este proceso, de lo que estamos hablando es de emancipación en el marco de la transición a la edad adulta. 
Por todo ello, la emancipación pasaría a considerarse como un proceso de integración en que se dejan atrás responsabilidades, y se inicia la aceptación y el desarrollo de nuevos roles asociados a nuevas responsabilidades, las responsabilidades asignadas al adulto. Así podríamos hablar del destino social de ser adulto como integración (Garrido y Requena, 1997: 15). Según este punto de vista, estaríamos en una fase de la vida en la que se establecen formas de vinculación y participación dentro de la organización social, lo que hace que los jóvenes se incorporen como personas sociales (Ibídem, 15). Si este proceso no se da, las consecuencias de desestructuración social son más que evidentes y por tanto, estaríamos más cerca de la exclusión social que de la inclusión y, con ello, de personas que podrían estar dejando de participar como ciudadanos en el mundo de los adultos.

La no emancipación de los jóvenes españoles en condiciones que les permita la transición a la edad adulta alumbra la idea de la polaridad conceptual de la que nos habla Tezanos (2004:12) al referirse a la dualidad excluido - incluido. Es decir, si aceptamos que un joven que no se emancipa puede caer en exclusión estamos indicando que lo será de otro mundo ya incluido. En definitiva, estudiar la exclusión por la vía de la emancipación nos obliga a conocer cuáles son los factores y, con ello, los recursos que consideramos definitorios de la inclusión. Algo que vamos a tratar de presentar en este artículo.

\section{LOS RECURSOS DE LA EMANCIPACIÓN}

Una pregunta que tenemos que hacernos es si los jóvenes españoles tienen o no claro qué es estar emancipado. Con datos del CIS y su estudio 2733 (2007) en el marco de los Sondeos de la Juventud Española podemos responder a esta cuestión y veremos cómo las respuestas parecen acordes con los planteamientos teóricos que manejamos, siendo la emancipación mucho más que no depender de los padres y/o familia de origen (Tabla 1).

Tabla 1. ¿Qué significa para los jóvenes estar emancipado?

\begin{tabular}{|l|c|}
\hline Respuesta & Porcentaje (\%) \\
\hline Vivir solo, irte de casa de tus padres & 36,4 \\
\hline Ser independiente económicamente & 25,3 \\
\hline Tener tu propia familia & 1,3 \\
\hline Adquirir responsabilidades & 3,3 \\
\hline Hacer lo que quieras, ser libre & 7,5 \\
\hline Otras Respuestas & 0,5 \\
\hline No Sabe & 24,2 \\
\hline No Contesta & 1,5 \\
\hline
\end{tabular}

Fuente: Elaboración propia a partir del fichero de microdatos del "Sondeo sobre la juventud española" - Estudio 2733 (2007) del Centro de Investigaciones Sociológicas (www.cis.es).

Estar emancipado sí es descrito por los jóvenes como algo más que vivir fuera de la casa de los padres - abandonar el hogar de la familia de origen. Según los datos del Estudio 2733 del CIS, para el 36,4\% de los encuestados es "vivir solo, irse de la casa de los padres". Junto al hecho de abandonar el hogar familiar como aspecto descriptor del significado de emancipación, tendríamos en segundo lugar el hecho de "ser independiente económicamente" (el porcentaje alcanzado aquí es del 23,3\%). En este caso, se puede dar la circunstancia de poder ser encuadrado entre el segmento joven que es independiente en términos económicos y aún sigue viviendo en el hogar familiar de origen. Se consideran 
emancipados pero no estarían siendo registrados por las tasas de emancipación. También aparece como factor explicativo de la emancipación la posibilidad de "hacer lo que se quiera" $(7,5 \%)$, "adquirir responsabilidades" $(3,3 \%) \mathrm{y}$, finalmente, entre esas acepciones del concepto estaría el "formar una familia" $(1,3 \%)$. Más allá de esta forma de definirlo tendríamos que señalar un hecho más que llamativo: el 24,2\% de los jóvenes no es capaz de definir el concepto, no saben qué es, siendo jóvenes que, al ser preguntados, no son capaces de explicarlo. En todo caso, vemos como son muchos los factores que definirían la emancipación y, con ello, muchos los recursos que deben manejar los jóvenes para alcanzarlos. En todo caso, lo que sí parece evidente, es que una mayoría de jóvenes tiene más o menos claro qué es estar emancipado.

Si adoptamos la perspectiva que venimos definiendo, interpretaremos, por tanto, la emancipación como proceso de integración social en un mundo adulto, como una transición al mundo adulto. Pero para poder llevar a cabo esa integración social son necesarios una serie de recursos, seguir un proceso de adquisición de recursos que Garrido y Requena (1997:1719) resumen, entre otros, en: actividad productiva en el marco de la inserción laboral y con ello recursos económicos, y residencia propia. Veamos con brevedad algunos aspectos relacionados con estos recursos.

\subsection{La actividad productiva laboral como factor de independencia económica}

Como ya venimos indicando, entre las formas de independencia propias de los adultos que señalan Garrido y Requena (1997), la clave fundamental, desde nuestro punto de vista, estaría en la inserción laboral que viene de la mano del proceso de acceso al mercado laboral en condiciones de estabilidad y seguridad, forma ésta a la que cada vez los jóvenes acceden más tarde y en peores condiciones.

Este proceso de acceso al mercado, como indican García Blanco y Gutiérrez (1996:269), suele coincidir, en la mayoría de las sociedades, con la etapa juvenil, y viene a consistir en "una transición social que va desde las posiciones en el sistema educativo y la familia de origen, hacia posiciones de mercado y de emancipación e independencia familiar".

Sin embargo, en el caso de España, y en concreto para referirnos a los jóvenes y el tipo de empleos en los que se inician en el mercado laboral, lo que encontramos es la idea de trabajo atípico como aspecto propio de una sociedad como la nuestra, una sociedad contemporánea, en la que cada vez existe "mayor persistencia y, a menudo, crecimiento de formas de trabajo atípico, trabajo que se define mejor por lo que no es que por lo que es: un sinnúmero de formas de trabajo que se apartan de la norma" (Rodgers y Rodgers, 1992:16) como son todas las modalidades existentes de contrato temporal, la eventualidad, la parcialidad en la jornada de trabajo, etc. Formas de trabajo propias de los jóvenes, especialmente en sus primeros empleos. Esto termina definiendo procesos de transición laboral que se alargan en el tiempo, alejados de la seguridad y de la estabilidad.

De esta forma se inicia un proceso en el que el mercado de trabajo se segmenta y la fuerza de trabajo se termina bipolarizando, en lo que se han venido a llamar los mercados de trabajo duales. Doeringer y Piore (1983:310-311) señalan que el enfoque del mercado dual del trabajo entiende éste como segmentado en un sector primario y en un sector secundario. Dentro del primero estarían aquellos puestos de trabajo con mejores salarios, con mayor estabilidad y más valorados y destacados en la sociedad. Aquellos que forman parte de esta parte del mercado laboral tienen más seguridad en el empleo y mayores posibilidades de promocionar. En cambio, el sector secundario, o mercado laboral secundario, se caracterizaría por empleos mal pagados, con alto grado de inestabilidad y sin posibilidades, al 
menos en teoría, de avanzar, de promocionar. Aquellos trabajadores que definen trayectorias laborales en este segundo sector viven frecuentes suspensiones en el empleo, entradas y salidas del mercado laboral. Este es el caso de los jóvenes españoles que, como afirmaba Casal Ballater (1996), describen cada vez con mayor frecuencia trayectorias de aproximación sucesiva y obreras, con frecuentes entradas y salidas del mercado laboral y con alto grado de inestabilidad e inseguridad en los momentos en los que están insertos laboralmente.

Esta falta de continuidad laboral y, con ello, falta de estabilidad laboral, queda explicada en el momento en el que descubrimos cuántos contratos han tenido los jóvenes a lo largo de su vida laboral (Tabla 2).

Tabla 2. Distribución del número de contratos remunerados: los jóvenes en España (\%)

\begin{tabular}{|l|c|}
\hline $\mathrm{N}^{\mathbf{o}}$ de contratos & Porcentaje (\%) \\
\hline Uno & 12,3 \\
\hline Dos & 19,6 \\
\hline Tres & 22,3 \\
\hline Cuatro & 15,4 \\
\hline Cinco o más & 25,5 \\
\hline No sabe / No contesta & 4,9 \\
\hline
\end{tabular}

Fuente: Elaboración propia a partir del capítulo II "Economía, empleo y consumo" del Informe de la Juventud en España (2008), INJUVE, Ministerio de Igualdad.

El Informe de la Juventud en España del INJUVE (2008) viene a confirmar que existe un alto índice de salidas y entradas del mercado laboral por parte de los jóvenes españoles o, lo que es lo mismo, trayectorias de inserción laboral profundamente desestructuradas. Poco más del 10\% afirma haber tenido un único contrato de trabajo. Entre dos y tres empleos dice haber tenido más del $40 \%$ de los jóvenes. Cuatro un 14,5\%. Y, finalmente, cinco o más contratos lo señala más de una cuarta parte de la muestra analizada en este estudio. Estos datos confirman altas tasas de temporalidad en el empleo juvenil en España. Confirman frecuentes entradas y salidas del mundo del trabajo. Y son, así mismo, signos de inestabilidad laboral, inseguridad y ejemplo de flexibilidad del mercado laboral en el caso de los jóvenes que comienzan el proceso de inserción laboral y, con ello, de emancipación e inserción social.

La aspiración a ocupar el lugar del adulto en la estructura social se desvanece en este escenario de inestabilidad y más cuando lo que los jóvenes buscan en un trabajo es, fundamentalmente, la seguridad y la estabilidad. En concreto, el 38,7\% de los jóvenes entrevistados por el CIS en el Sondeo de la Juventud Española - Estudio 2631 (2006) afirma que, a la hora de valorar un empleo, lo que para ellos es clave es la seguridad y la estabilidad. En suma, el desajuste de expectativas es más que notorio. Lo que desean, no lo encuentran. Esto queda resumido de forma clara cuando, en las entrevistas en profundidad, se les pregunta por cuál es el valor del trabajo en las sociedades actuales. El trabajo es interpretado como un elemento básico de la sociedad en la que vivimos. Se interpreta como el motor que mueve a toda la sociedad. No se entendería la sociedad actual si no existiera el elemento del trabajo:

"Para mover el mundo. Para todo, para la familia, para la economía, para comprarte

la casa, para todo... el trabajo es el motor económico de un país”. (E6, mujer, 27)

El trabajo se vive como experiencia que aporta satisfacción ante sí mismos y ante la sociedad en la que están inmersos, y todo ello en términos de utilidad en tanto que parte funcional de la sociedad en la que se encuentran insertos. Además, el trabajo es una forma de 
demostrarse que pueden comenzar a vivir su independencia, y un medio a través del cual lograr aquellos aspectos materiales que se consideran objetivos vitales y, todo ello, en un marco de realización personal:

"Trabajar claro que es importante porque te sientes útil. Te sientes útil con la sociedad, te sientes útil tú como persona, te sientes bien por dentro, no sé...Sabes que te vales por ti mismo.... eso..." (E12, mujer, 40)

"Y... pero básicamente el trabajar es el medio primero que te realiza, para poder conseguir las metas que tú te has establecido” (E1, hombre, 36)

Se habla de emancipación en términos laborales, lo que implica la posibilidad de estar inserto laboralmente en el mercado de trabajo y, con ello, la posibilidad de abandonar el hogar familiar de origen mediante la adquisición de la propia vivienda (aspecto este que trataremos más adelante). El factor económico aparece como el principal determinante de las posibilidades de emancipación. Si no se tienen los recursos necesarios, difícilmente se puede aspirar a la emancipación, de forma que el empleo como medio aceptado para la obtención de los ingresos necesarios se convierte en uno de los requisitos básicos de emancipación. Comparándose con la vida adulta normalizada en la que se posee todo lo indicado es, por tanto, como definen los jóvenes e interpretan lo que es la emancipación:

"Estar emancipado es dejar de depender de los padres, pero no sólo económicamente

[...], es formar tu propia familia, tener trabajo, tener tu piso, ...” (E8, hombre, 4)

"Si no te emancipas es que no tienes dinero, y si no tienes trabajo...pues no tienes dinero y asi no se puede” (E6, mujer, 3)

Por tanto, hablar de la adquisición de una actividad productiva supone la adquisición de una ocupación en el mercado laboral. Pero no puede ser un empleo cualquiera, debe ser un empleo remunerado con la finalidad de poder conseguir bienes y servicios del mercado de forma independiente. Es decir, un nivel salarial mínimo para asegurar el paso al mundo adulto, sin dependencias familiares y con capacidad económica propia. Hoy por hoy, y habría que analizarlo en mayor profundidad, algo que no puede ser objeto de este artículo, parece un terreno vedado para una parte importante de la población joven.

\subsection{La vivienda propia como factor de emancipación}

El retraso en la edad de emancipación de los jóvenes españoles, asociado inevitablemente al retraso de la inserción laboral plena, es uno los rasgos que caracteriza a este segmento de población, y es uno de los aspectos por los que el interés por su estudio sigue vigente. Pero hay otras razones igualmente importantes. Entre esas otras que explicarían dicho retraso, más a allá de la escasez en la oferta de puestos de trabajo, estaría en la dificultad de acceso a la vivienda (Leal Maldonado, 2002:249). En este sentido, estarían faltando dos de los aspectos básicos señalados anteriormente en relación con la integración: adquisición de una ocupación $\mathrm{y}$ adquisición de una vivienda propia.

Los jóvenes que se integran al mundo adulto lo hacen mediante un hogar o residencia diferente al de la familia de origen, como capital físico que permite distanciarse de las dependencias respecto a ésta. La no adquisición de este recurso es y debe ser considerada como un elemento que permita hablar de transición a la vida activa y transición a la vida adulta incompleta, en tanto no cumple una de las condiciones necesarias para hablar de independencia. La obtención de esta adquisición necesita del primer elemento ya analizado: un empleo remunerado. Sin empleo remunerado a un determinado nivel y sin vivienda propia 
al margen del hogar familiar de origen nos encontramos ante una disfunción clara del proceso de emancipación y, con ello, de la idea de ciudadano plenamente integrado.

En esta misma línea se expresa Jurado Guerrero (2003:113), quien viene a confirmar que los problemas de acceso a la vivienda están detrás de las dificultades que se observan en España de cara a la formación de una familia, donde el mercado de viviendas en nuestro país, incluso la propia regulación de dicho mercado, favorece sobre todo a las familias insertadas laboralmente, mientras que serían los mercados de acceso relativamente fácil a la vivienda en alquiler los que podrían favorecer la formación de lo que, ella misma denomina, familia temprana. Este segundo tipo de mercado se adaptaría mejor a las familias jóvenes que tienen un mayor carácter de innovación y prueba. Sin embargo, como bien sabemos, sin empleo tampoco se tienen los ingresos necesario para optar por este mercado de vivienda de alquiler.

Según datos del Informe de la Juventud en España del INJUVE (2008) a casi 3 de cada 10 jóvenes españoles de entre 15 y 29 años, les gustaría poder comprarse una casa y no pueden hacerlo. Todos conocemos y hablamos de las dificultades con que cuenta la población joven para acceder a esa vivienda deseada, dificultades que se mantienen aunque ésta sea en alquiler, pero los datos lo dejan todo aún más claro.

En concreto y como demuestran los datos del OBJOVI (Observatorio Joven de la Vivienda en España) del Consejo de la Juventud en España, presentando los parámetros de acceso a la vivienda (en propiedad o alquiler), y refiriéndonos al segundo trimestre de 2009, el precio medio de una vivienda libre en euros ascendía a los 192.090 euros, siendo el máximo tolerable por una persona joven de 92.415,27 euros. La única opción que quedaría sería acceder a la vivienda en alquiler. En el caso de la vivienda en alquiler, la renta media ascendía a 676,4 euros, siendo la renta máxima tolerable para alquiler de una persona joven de 376,06 euros. Creemos que los datos hablan por sí mismos y no requieren de mayor explicación.

En las entrevistas en profundidad, el no poder acceder a una vivienda en propiedad, y, en muchas ocasiones, ni siquiera a una en alquiler, es una razón para no abandonar el hogar familiar. El discurso está bastante consensuado al respecto, pero también actúa como factor de desajuste de expectativas. Con ello nos estamos refiriendo a que los deseos de emancipación temprana se ven insatisfechos ante la imposibilidad de acceder a una vivienda, factor éste que estaría asociado al tipo de empleo, precario y de bajo sueldo, con el que suelen empezar sus trayectorias laborales los jóvenes:

P: ¿Podías haberte independizado antes?

R: "Eh, no, lo estuve intentando pero no pude (risas) No, yo me hubiera independizado, a los dieciocho años ya di la primera alarma de que me independizaba, sí, pero por que ... bueno, yo me quería independizar, pero claro yo quería, bueno, comprarme mi piso, y tal. Y a esta edad pues no tuve dinero para hacerlo". (E10, mujer, 21)

"Porque no me puedo comprar una casa. A veces he pensado en alquilarme algo para irme pero la verdad es que me molesta eso de tener que pagar lo mismo que si fuera una hipoteca" (E1, hombre, 19)

Por tanto, las expectativas de emancipación vía vivienda, también se ven recortadas cuando no completamente amputadas. Así, este factor esencial de emancipación tampoco se da como debería darse y, con ello, se dificulta aún más la emancipación y la transición al mundo adulto. 


\section{DEPENDENCIA VERSUS INDEPENDENCIA}

El proceso de integración de los jóvenes en el mundo de los adultos concluye cuando se han abandonado las dependencias originarias familiares. En este sentido, parece razonable suponer que la obtención y asimilación de esos elementos analizados y, por tanto, el proceso de adquisición de éstos, es lo primero que debemos tener presente si queremos entender cómo se produce el citado abandono. (Garrido y Requena, 1997:17). Si esto no se alcanza, no por falta de interés del joven, sino por falta de oportunidades, el proceso se alarga, se ralentiza, cuando no se eterniza, como puede estar pasando en la actualidad en España. Las dependencias nunca finalizan y las independencias pueden adquirir la forma de deseo inalcanzable, lejos de la mano, deseo que no es sino eso, un deseo.

Esto nos lleva a la idea de que en las últimas décadas, y de manera progresiva, se ha ido dificultando la transición a la vida laboral (algo de lo que ya hemos hablado y conocemos bien), y con ello, se ha dificultado la adquisición de la independencia necesaria para la emancipación (Navarro López, 2004:379). Se trata este de un problema que afectaría no sólo a los jóvenes, sino a sus familias y a la sociedad en general. En cambio, parece existir consenso social en aceptar como un hecho normal que se extienda en el tiempo este proceso de inserción laboral, lo que trae consigo la posposición de la emancipación (Ibídem, 379). En definitiva, estamos ante un proceso cuyas fronteras de fin parecen poco claras, cuando no inquebrantables.

Tabla 3. Autonomía de gasto de los jóvenes en España - 2008 (\%)

\begin{tabular}{|l|c|}
\hline Situación de dependencia - independencia & Porcentaje (\%) \\
\hline $\begin{array}{l}\text { Puede pagar todos los gastos (propios y/o pareja) incluso vivienda y } \\
\text { alimentación - PLENAMENTE INDEPENDIENTES }\end{array}$ & 35,5 \\
\hline $\begin{array}{l}\text { Puede casi todos los gastos (propios y/o pareja) pero no todos - } \\
\text { SEMI INDEPENDIENTES }\end{array}$ & 24,3 \\
\hline $\begin{array}{l}\text { Sólo puede pagar los gastos de bolsillo (propios y/o pareja) - } \\
\text { VIVEN O DEPENDEN DE LOS PADRES }\end{array}$ & 40,2 \\
\hline
\end{tabular}

Fuente: Elaboración propia a partir del capítulo II "Economía, empleo y consumo" del Informe de la Juventud en España (2008), INJUVE, Ministerio de Igualdad.

A este respecto y si nos detenemos en los datos de la Tabla 3 vemos que la mayoría de los jóvenes españoles no son independientes plenamente. Es decir, por un lado el 40,2\% vive o depende de los padres, de tal forma que sólo pueden pagar sus gastos de bolsillo, los gastos en ocio y tiempo libre.

A ellos habría que sumar otro $24,3 \%$ que tampoco es plenamente independiente, de hecho son conceptualizados como semi-independientes en tanto pueden pagar casi todos los gastos pero no todos, o lo que es lo mismo, necesitan apoyo externo. Finalmente contaríamos con los plenamente independientes que, en el estudio del INJUVE (2008), se corresponden con el $35,5 \%$ de los jóvenes, jóvenes que con sus ingresos propios pueden hacer frente a todos los gastos incluso los derivados de la vivienda y la alimentación, cifra esta última que, con seguridad, en los últimos años habrá bajado considerablemente.

Pero, ¿comprendemos bien el concepto de dependencia y de independencia? El concepto dependencia alude a la cobertura de ciertas necesidades básicas por parte de una entidad -sea esa entidad individual o colectiva-, entidad que no coincide con el elemento dependiente. Es decir, un individuo "depende de la persona o colectividad que proporciona los bienes y servicios o las relaciones que satisfacen las necesidades que están en el origen de la 
dependencia" (Requena, 2002:21). Además, a medida que aumenta el valor o coste de los servicios o productos que el grupo provee internamente más dependiente es el individuo (en nuestro caso el joven) del grupo (en este caso, de la familia). En este hecho, según Requena, (Ibídem, 22) estaría la explicación de las dificultades de emancipación. Es decir, "si lo que le proporciona su familia se puede conseguir con menos coste fuera de casa, su grado de dependencia familiar" será más bajo que si lo que le proporciona la familia tiene un alto coste fuera de ella. Por tanto, cuánto más coste tenga la independencia, mayor dependencia familiar. Sin duda, esta sería la hipótesis que debemos manejar en el caso de España: los jóvenes españoles, no por falta de "ganas", sino por falta de escenarios propicios, prefieren depender de sus familias que buscar una independencia costosa, plagada de incertidumbre, actuando así la familia como marco de tranquilidad.

Así, el grado de dependencia familiar estará mediado tanto por lo que suceda dentro del grupo familiar como lo que suceda fuera. En relación a lo primero, a la dependencia familiar mediada desde dentro de la familia, deberemos analizar qué transformaciones familiares pueden modificar el balance que hacen los jóvenes entre aquello a lo que les obliga la familia y lo que la familia les proporciona. En el caso de la perspectiva externa nos referimos a las modificaciones o alteraciones de los costes de las alternativas a los bienes y servicios que se consiguen en la familia, en tanto elementos que reducen o aumentan la posibilidad de acceder a ellos fuera del hogar de origen (Requena, 2002:22). Por tanto, estaríamos dentro de un proceso de elección racional y de toma de decisión sobre la base del estudio tanto de la oferta interna como la externa a la familia, proceso que requiere, por parte de los jóvenes, encontrar en la inserción laboral y en la emancipación un valor de realización personal.

En nuestro trabajo en general, y en este artículo en particular, partimos de que la inserción laboral es básica para la inserción social, todo ello enmarcado como factor de independencia. Así, y respecto a la importancia social de la adquisición de una ocupación como elemento de inserción laboral, nos parecen muy acertadas las consideraciones de Serrano Pascual (1995: 179-180) quien considera que, partiendo de que en las sociedades contemporáneas el valor de la reciprocidad es clave de cara al propio mantenimiento de dichas sociedades, la inserción laboral actuaría como fuente de reconocimiento y como fuente de reproducción social, todo ello marcado por la posibilidad de la independencia, frente a la dependencia originaria.

Si la sociedad no le da al joven la posibilidad de trabajar, el joven no puede devolver a la sociedad aquello que le ha aportado, no podría reproducirse dicha sociedad. Los jóvenes aparecerían de esta forma asociados a un estado deficitario. En este sentido, si los jóvenes no se insertan laboralmente perderían parte de la legitimación pública que la inserción laboral supone. Así, el trabajo, la inserción laboral, actuaría como factor de autonomía y de afirmación de valor supremo de la persona, que vendría de la mano del reconocimiento del joven como activo de la sociedad, como miembro adulto de la sociedad. "La inserción laboral interviene así como condición y consecuencia de la afirmación de la potencia humana que fundamenta en gran medida el principio de la modernidad" (Serrano Pascual, 1995:180). En definitiva, hablamos del ciudadano.

Por eso, y según se desprende del discurso analizado en las entrevistas en profundidad, el empleo es clave, casi como primera piedra necesaria para la emancipación, para dejar de depender y ser independiente. El trabajo se vive como experiencia que aporta satisfacción ante sí mismos y ante la sociedad en la que están inmersos, y todo ello en términos de utilidad en tanto que parte funcional de la sociedad en la que se encuentran insertos. Eso sí, siempre y cuando ese empleo sea seguro y estable. Además, el trabajo es una forma de demostrarse que pueden comenzar a vivir su independencia, y un medio a través del cual 
lograr aquellos aspectos materiales que se consideran objetivos vitales y, todo ello, en un marco de realización personal:

"Trabajar claro que es importante porque te sientes útil. Te sientes útil con la sociedad, te sientes útil tú como persona, te sientes bien por dentro, no sé...Sabes que te vales por ti mismo.... eso..." (E12, mujer, 40)

"Y... pero básicamente el trabajar es el medio primero que te realiza, para poder conseguir las metas que tú te has establecido” (E1, hombre, 36)

Tener éxito o fracaso en el mundo del trabajo contribuye a que los jóvenes desarrollen su propia identidad en su paso a la edad adulta y, a través de ella, contribuyan a la reproducción de la sociedad. De esta forma, las trayectorias de acceso al mercado laboral y cómo éstas se desarrollen (Casal Ballater, 1996) se verán influidas por el contexto social y económico que se viva en cada momento. En este sentido, la actual situación de crisis económica está afectando de forma más directa a los más jóvenes. Esto puede estar generando un proceso de desmotivación hacia su futuro, llegando a ver el paro casi como algo inevitable, del que difícilmente se podrá salir y más si se es joven. Esto podría poner en riesgo las transiciones a la edad adulta de muchos jóvenes españoles que experimentarían desajustes entre deseosexpectativas y la realidad del mercado laboral juvenil.

Uno de los colectivos que podría ver más afectado el valor del trabajo en tanto que fuente de identidad puede llegar a ser, más que el de los que aún no han salido del hogar familiar, los que en los últimos años habían abandonado el hogar origen y habían comenzado a realizar su transición a la vida adulta desde el punto de vista de la emancipación familiar y la independencia económica. En este caso, una posible vuelta al hogar familiar rompería el proceso de transición vía mercado laboral y se transformaría en un ejemplo de ruptura con la norma social, fruto del desempleo y la inseguridad laboral. Nos encontramos así con jóvenes que se están planteando volver a casa de los padres al haber quedado en paro y no poder hacer frente a la, en muchos casos, recién estrenada independencia:

"Yo llevaba viviendo 2 años en mi propio piso, era alquilado y eso... pero me veo volviendo con mis padres si la cosa no cambia” (E15, hombre, 4)

También nos encontraríamos con jóvenes que pueden ver mermada su identidad personal como trabajadores si, habiendo estado insertos en el mundo laboral, viven situaciones de desempleo. Por ello, la independencia económica como valor personal alcanzado se pone en duda en el discurso y se activa aún más el valor del trabajo como fuente de identidad personal:

"No, para mi es muy importante, yo es que es lo que te comentaba antes, he sido muy independiente, y yo todo lo que tengo me lo he ganado yo, y que me gusta ganarme las cosas, no me gusta que nadie me dé nada. Eh, el trabajo también es muy importante, porque, por ejemplo, ahora mismo no estoy trabajando, pero hasta ahora, he estado cobrando el paro, pero ahora llevo ya una semana sin cobrar el paro, y eso de no ganar yo el dinero, pues me siento muy rara” (E10, mujer, 28)

\section{CONCLUSIONES}

Albert (1998:4) señala de forma muy acertada que, una vez los jóvenes han finalizado su periodo formativo, lo hayan hecho con éxito o no, pueden tomar, fundamentalmente, dos 
direcciones: o bien dirigirse a la actividad, o hacerlo hacia la inactividad (tanto si están desempleados como ocupados). Estas dos trayectorias no causarían incertidumbre en los jóvenes si no fuera por las dificultades que estos tienen de cara a la inserción laboral, si la opción de entrar al mercado fuera eso, una opción y no una barrera.

Esas dificultades no sólo suponen un coste para el joven en el plano personal, sino que también tienen un importante coste social, convirtiéndose esto en un tema de profunda preocupación tanto para los poderes públicos, como para el propio mercado laboral y para el sistema educativo. Estos últimos como responsables de la formación, el mercado laboral como espacio en el que tiene que desarrollarse el proceso de inserción laboral y responsable de la demanda de trabajo, y los poderes públicos por ser los que deben desarrollar las políticas de intervención más ajustadas a las necesidades de inserción de los jóvenes (las políticas activas de empleo). No es necesario que redundemos en que esta triple conjunción pues, hoy por hoy y en la actual situación, no se da, al menos en los términos en que sería deseable de cara a la inserción socio - laboral de los jóvenes en España.

En definitiva, hablar de emancipación y de inserción social no siempre resulta fácil, son muchas las aristas que estos conceptos implican, pero sí hemos podido encuadrar mejor, con la brevedad de esta aportación, el objeto de análisis en la que los procesos, estos dos procesos, son clave para entender las transformaciones en la transición a la edad adulta de los jóvenes españoles, marcada por las dificultades, los desajustes de expectativas y un disfuncional funcionamiento de la estructura social.

Estamos en un momento clave, y el tiempo apremia a las ciencias sociales para que se realicen estudios profundos sobre lo que está sucediendo con la juventud española. Se hace necesario profundizar de forma clara en todos estos procesos, delimitar bien los escenarios y realizar propuestas tanto para el sistema educativo, como para el mercado y el Estado. Los cambios que se deben poner en marcha han de ser inevitablemente estructurales. Si en períodos de bonanza económica las tasas de desempleo juvenil son altas es porque ese ajuste no se da o, si se ha intentado no ha ido en la dirección correcta. Si en períodos de crisis económica, como la actual, dichas tasas se disparan es porque puede que nuestro sistema productivo no esté adaptado a nuestro capital humano.

En todo caso, lo que sí parece claro es que los jóvenes quieren ser adultos, quieren comportarse como adultos, desean ser adultos. Sin embargo, parece como si el mundo de los adultos no les permitiera y/o no pusiera las condiciones para poder desarrollar ese rol social. En suma, puede que estemos sembrando la creación de un no-ciudadano, no integrado, no realizado. No cabe duda de que estamos ante un problema social que deberá ser analizado en años venideros, y compartido y discutido en la comunidad científica.

\section{BIBLIOGRAFÍA}

ALBERT, C. (1998), Las transiciones de los jóvenes de la escuela al mercado de trabajo: un análisis de flujos, Valencia: Working Papers - Instituto Valenciano de Investigaciones Económicas.

CASAL BALLATER, J. (1996), "Modos emergentes de transición a la vida adulta en el umbral del siglo XXI: aproximación sucesiva, precariedad y desestructuración", en Revista Española de Investigaciones Sociológicas, $\mathrm{n}^{\mathrm{o}}$ 75, pp. 295-316.

DOERINGER, P.B. y PIORE, M.J. (1983), "El paro y el mercado dual de trabajo", en Toharia, L. (coord.), El mercado de trabajo: Teorías y aplicaciones. Lecturas seleccionadas, Madrid: Alianza.

GARCÍA BLANCO, J.M. y GUTIÉRREZ, R. (1996), "Inserción laboral y desigualdad en el mercado de trabajo: cuestiones teóricas”, en Revista Española de Investigaciones Sociológicas, n 75, pp. 269-293 
GARRIDO, L. y REQUENA, M. (1997), "Emancipación y adquisición: las dos caras de la integración en el mundo adulto", en Garrido, L. (coord.), La emancipación de los jóvenes en España. Madrid: Ministerio de Trabajo y Asuntos Sociales, pp. 9-21

INJUVE (2008), Informe de la Juventud en España. Madrid: Ministerio de Igualdad.

JURADO GUERRERO, T. (2003), "La vivienda como determinante de la formación familiar en España desde la perspectiva comparada", en Revista Española de Investigaciones Sociológicas, $\mathrm{n}^{\circ} 103$, pp. 113-157.

LEAL MALDONADO, J. (2002), "Retraso de la emancipación juvenil y dificultad de acceso de los jóvenes a la vivienda" en VV.AA. (2002), La sociedad: teoría e investigación empírica. Estudios en Homenaje a José Jiménez Blanco, Madrid, Centro de Investigaciones Sociológicas - CIS, pp. 249-264. NAVARRO LÓPEZ, M. (2004), "Los jóvenes y la familia”, en ARBOR, nº 702. pp. 377-400.

PARSONS, T. (1998), "La estructura social de la familia", en Nanda Anshen, R., Fromm, Horkheimer, Parsons y Otros. La familia. Barcelona: Península, pp. 31-66.

REQUENA, M. (2002), "Juventud y dependencia familiar en España", en Revista de Estudios de Juventud, $\mathrm{n}^{\circ}$ 58, pp. 19-32.

RODGERS, G. y RODGERS, J. (1992), El trabajo precario en la regulación del mercado laboral. Crecimiento atípico en la Europa Occidental, Madrid: Ministerio de Trabajo y Seguridad Social.

SERRANO PASCUAL, A. (1995), "Procesos paradójicos de construcción de la juventud en el contexto de crisis del mercado de trabajo", Revista Española de Investigaciones Sociológicas, nº 71, pp. 177-199. TEZANOS, J. F. (2004), "Tendencias de dualización y exclusión social en las sociedades tecnológicas avanzadas. Un marco para el análisis", en Tezanos, J.F. (ed.), Tendencias en desigualdad y exclusión social. Tercer Foro sobre tendencias sociales, Madrid: Sistema, pp. 13-53.

\section{Breve currículo:}

\section{José Manuel García Moreno}

Doctor en Sociología por la Universidad de Granada. Premio Extraordinario de Licenciatura en Sociología por la Universidad de Granada y Primer Premio Nacional de Terminación de Estudios en Sociología por el Ministerio de Educación en 2001. Máster en Dirección de Márketing y Gestión Comercial en la Escuela de Negocios ESIC. Con amplia trayectoria profesional y empresarial en Comunicación Política. En la actualidad es profesor del Departamento de Sociología de la Universidad de Granada siendo sus campos de especialización la Sociología del Trabajo, la Sociología Política y las Técnicas de Investigación.

\section{Rafael Martínez Martín}

Profesor Titular de Universidad en la Universidad de Granada. Doctor en Ciencias Políticas y Sociología por la Universidad de Granada. Premio Extraordinario de Doctorado. Autor de varios libros y artículos en revistas científicas sobre las temáticas de mercado de trabajo e inserción profesional. Ha trabajado como profesor invitado en el École des Hautes Études en Sciences Sociales de París. En la actualidad sus líneas de investigación están centradas en mercado de trabajo y bienestar social. 